\title{
'Desirada': des voix contre le silence
}

\section{Anne Marie Miraglia}

\section{OpenEdition}

Journals

Édition électronique

URL : http://journals.openedition.org/studifrancesi/34088

DOI : 10.4000/studifrancesi.34088

ISSN : 2421-5856

\section{Éditeur}

Rosenberg \& Sellier

\section{Édition imprimée}

Date de publication : 1 novembre 2005

Pagination : 292-301

ISSN : 0039-2944

\section{Référence électronique}

Anne Marie Miraglia, «'Desirada': des voix contre le silence », Studi Francesi [En ligne], 146 (XLIX | II) | 2005, mis en ligne le 30 novembre 2015, consulté le 20 avril 2021. URL : http://

journals.openedition.org/studifrancesi/34088; DOI : https://doi.org/10.4000/studifrancesi.34088

\section{(c) (i) (9)}

Studi Francesi è distribuita con Licenza Creative Commons Attribuzione - Non commerciale - Non opere derivate 4.0 Internazionale. 


\section{'Desirada': des voix contre le silence}

Cette étude vise l'exploration de la technique narrative utilisée dans Desirada (1997) de Maryse Condé. Notre objectif principal est d'analyser comment la polyphonie, ou la pluralité de récits et de voix narratives dans le texte, fait éclater le mensonge et le silence qui empêchent Mari-Noëlle, la protagoniste, de s'épanouir. En se mettant à l'écoute de voix autres que celle de sa mère Reynalda, Marie-Noëlle arrive à se créer une vérité et une identité qui lui sont propres et sans lesquelles il lui est difficile «d'aller de l'avant» et de réaliser son rêve d'écriture. Cette étude examinera donc, en dernier lieu, le lien entre la polyphonie du texte et la thématique de l'écriture.

Tout d'abord, l'aspect polyphonique de Desirada participe d'une grande richesse esthétique qui relève d'un processus de multiplication présent non seulement au niveau narratif (multiplicité de voix et de récits), mais aussi sur les plans thématique, temporel et spatial. On y trouve un va-et-vient constant entre le réel et l'imaginaire ainsi qu'entre le passé et le présent, un contrepoint s'effectuant principalement à travers trois pôles géographiques distincts: la Guadeloupe (La Pointe, Basse-Terre, la Désirade), la France (Paris, Nice), et les États-Unis (Boston). Le texte touche ainsi à plusieurs thèmes récurrents dans l'écriture de Maryse Condé et de grande actualité dans le village global d'aujourd'hui: l'exil, l'errance, l'immigration, le racisme, l'hybridité culturelle et, bien sûr, la question primordiale des identités individuelle et collective dans un monde flou et en mouvement.

Le roman porte essentiellement sur la quête identitaire de Marie-Noëlle, une jeune femme métisse née en Guadeloupe d'une mère célibataire de 16 ans (Reynalda Titane) qui l'abandonne à sa naissance pour refaire sa vie en métropole. Dix ans plus tard, Reynalda arrache la petite fille à l'amour incommensurable de Ranélise Tertullien (sa 'mère adoptive') pour l'amener vivre en France mais sans pour cela lui témoigner le moindre signe de tendresse maternelle. Souffrant de tuberculose et profondément blessée par l'indifférence de sa mère, Marie-Noëlle quitte Paris à l'âge de 16 ans pour se faire soigner à Vence. A Nice, elle rencontre un jeune musicien, Stanley Watts, qui l'encourage à partir avec lui à Boston et qui l'épouse afin de lui faciliter l'immigration aux Etats-Unis - «le seul pays où un nègre peut réussir» ${ }^{1}$. Mais en Amérique, comme en France, Marie-Noëlle est incapable de mener à bien sa vie tant qu'elle n'aura pas dévoilé ses origines et l'identité de son père - «un homme à peau claire» à en juger par la blancheur de la peau de Marie-Noëlle (p. 152), par sa «tignasse jaune paille» et par «ses yeux que la lumière tigrait selon les moments en vert ou en jaune» (p. 34). Elle a 28 ans et est veuve depuis très peu de temps quand la mort de Ranélise lui fournit l'occasion de retourner en Guadeloupe et de mener une enquête sur l'identité de son père et sur sa propre conception. C'est là qu'elle rencontre pour la première fois sa grand-mère, Nina Titane qui l'ébranle avec un récit démentant les paroles de sa fille unique, Reynalda qui prétendait avoir été violée par l'amant de sa mère, le bijoutier italien Gian Carlo Coppini. Donc, loin de confirmer ses conjectures, ces trois semaines au pays natal ne font qu'élargir l'énigme sur ses origines et Marie-Noëlle rentre aux Etats-Unis encore plus troublée qu'à son arrivée. Elle poursuivra son enquête une année plus tard à Paris en interviewant son demifrère, Garvey, et surtout Mme Duparc qui avait facilité l'installation de Reynalda en France en lui offrant un poste de bonne pendant quatre ans. Marie-Noëlle poursuit son enquête en Belgique où elle va voir Ludovic qui a vécu 20 ans auprès de Reynalda

(1) Maryse Condé, Desirada, Paris, Éditions Robert Laffont, 1997, p. 78. A l'avenir toutes les citations de ce roman renverront à cette édition. 
avant d'être repoussé, lui aussi, comme tant d'autres «qui l'embarrassaient ou qui ne lui servaient plus à rien» (p. 269).

L'enquête de Marie-Noëlle fait éclater l'unité du récit en de multiples récits entendus à des moments différents de sa vie et dans les divers lieux géographiques où elle a vécu. Or nous verrons ici que cette multiplicité de voix et de récits constitue essentiellement une tentative de braver Reynalda - obstacle majeur à l'épanouissement de Marie-Noelle: «C'était à cause de Reynalda si elle n'avait de goût pour rien, pour personne si elle dérivait sans but dans l'existence» (p. 96). L'intégration de ces récits susceptibles de contredire (ou de confirmer) «la vérité» de Reynalda sera génératrice d'une écriture permettant à la fille de l'emporter sur sa mère pour la toute première fois.

Le roman se divise en trois parties qui suivent l'axe chronologique de la vie de Marie-Noëlle tout en opérant de nombreuses incursions, sous forme d'analepses, dans sa vie passée.

Ces analepses permettent la co-existence d'un espace concret, actuel, occupé physiquement par Marie-Noëlle et de l'espace abstrait de ses rêves, de son imagination et de récits entendus et remémorés. La première partie porte sur les 28 premières années de sa vie: Marie-Noëlle a vécu à la Guadeloupe jusqu'à l'âge de 10 ans, en France jusqu'à 20 ans et à Boston jusqu'à 28 ans. La deuxième et la troisième partie du texte sont structurellement identiques. Dans la deuxième partie, Le Récit de Nina, comprenant 21 pages (pp. 183-203), s'intercale entre les chapitres 5 et 6 tandis que dans la troisième partie, c'est Le Récit de Ludovic qui sépare le chapitre 5 du chapitre 6. Il importe surtout de signaler que c'est uniquement dans le tout dernier chapitre du roman que Marie-Noëlle deviendra narratrice à son tour. Cette promotion à la parole au style direct signale le commencement d'une certaine maîtrise que Marie-Noëlle n'acquiert que par le biais de l'écriture. De plus, il est évident que les voix narratives autonomes dans Desirada proviennent des trois personnages qui confèrent à Reynalda son statut de fille (Récit de Nina), d'épouse (Récit de Ludovic) et finalement de mère (Récit de Marie-Noëlle). Quant aux paroles de Reynalda ellemême, elles seront présentées par intervalles et uniquement à travers les souvenirs de sa fille.

Lorsque Marie-Noëlle retourne à la Guadeloupe en quête de certitudes sur ses origines, c'est à l'île de la Désirade qu'elle entendra le récit qui fera de Reynalda «cette menteuse de première» (p. 203) et qui opposera une autre «vérité» à celle que Reynalda avait confiée à sa fille:

Un jour, tu te rappelles, j'ai commencé à te raconter mon histoire. Je n'ai pas eu la force d'arriver jusqu'au bout parce que mes paroles me déchiraient la gorge. Je vais essayer de continuer en te passant les détails. Sinon, tu croiras que j'en rajoute. C'est tout ce que je peux te donner. La vérité. Dans l'espoir que tu comprendras et, que de cette manière, tu commenceras à vivre ta vie (pp. 101-102).

Effectivement, l'île de la Désirade fait à plusieurs reprises l'objet des cauchemars de Marie-Noëlle (pp. 145, p. 153, 159, 165) lors de son retour en Guadeloupe. Ainsi, en plus de donner au texte un titre espagnol (desirada, voir p. 62) et ironique (désir + en rade), cette île sert de leitmotif suggestif de l'abandon physique et des souffrances existentielles de l'enfant non désirée: et Marie-Noëlle et Reynalda (p. 190) sont les résultats d'un viol.

Si Marie-Noëlle arrive par moments à pleurer sur sa mère et à l'excuser de son indifférence, s'il lui arrive même des «bouffées de tendresse» (p. 105) à son égard, c'est surtout parce qu'elle prête encore foi au récit maternel relaté avant son départ pour Boston. Ainsi lorsqu'elle débarque en Guadeloupe 8 ans plus tard pour les fu- 
nérailles de Ranélise, c'est aussi avec «la mission précise» de «demander justice pour Reynalda» (p. 146) - qui violée et enceinte à 16 ans avait tenté de se suicider. Donc, la quête de Marie-Noëlle a comme objectif principal d'en savoir plus sur ses origines afin de pouvoir, elle aussi, «aller de l'avant»: «Bâtarde née de père inconnu. Belle identité que celle-là! Tant qu'elle n'aurait pas d'autres indications à inscrire sur son livret de famille, elle ne pourrait rien mener à terme» (p. 220).

Mais la réalisation de son projet s'avère difficile: Reynalda se referme sur son histoire car «les principaux acteurs de ce drame», l'évêque de la Guadeloupe et Gian Carlo Coppini, sont morts alors que le père Mondicelli et Fiorella (la fille de Gian Carlo et l'amie de Reynalda) sont hors d'atteinte. Marie-Noëlle sait que «si elle v[eut] connaître sa géographie et la carte de son identité, rien à faire, c'est sa grand-mère qu'il lui fa[ut] affronter» (p. 154). Ainsi lorsque Marie-Noëlle se présente à sa grandmère comme «la fille de Reynalda et... et de Gian Carlo», cette affirmation d'elle-même lui est bénéfique: «C'était la première fois qu'elle déclinait sa généalogie, qu'elle nommait au grand jour le nom de ceux qui l'avaient engendrée. Et c'était comme si enfin, elle prenait possession d'elle-même et qu'elle marquât sa trace sur terre.» (p. 180). Mais la réaction de Nina Titane - le rire - est dévastateur: «un rire qui d'un seul coup anéantissait les certitudes de Marie-Noëlle et la rejetait vers ce territoire du doute et de l'angoisse qu'elle avait cru quitter pour toujours» (p. 180).

Nommer ses parents c'est pour Marie-Noëlle l'affirmation de son existence et de son appartenance à un lieu précis et cela nonobstant sa bâtardise, son nomadisme et l'instabilité identitaire qui en résulte. Mais comme son rire, le récit que lui fait Nina ne fait que renvoyer Marie-Noëlle à son angoisse existentielle et ontologique:

Tu as l'air déçue, toute chagrinée. Ce n'est pas cette histoire-là que tu avais envie d'entendre, pas vré? Tu avais rêvé. Tu avais envisagé des tas d'autres choses dans ta tête et tu étais venue jusqu'ici, tu avais enjambé l'eau pour trouver un fondement à tes imaginations. Malheureusement, je ne peux rien t'offrir simplement pour faire plaisir à ton oreille. Je ne peux t'offrir que la vérité. Je ne peux te raconter que ce qui est arrivé. Gian Carlo n’a jamais été ton papa. Qui c'est? Seule Reynalda le connaît et peut te dire (p. 202).

Il faut remarquer que la voix de Nina est une voix autonome qui habite à elle seule tout un chapitre et s'oppose de plusieurs façons à celle de Reynalda. Plus brève, très fragmentaire et elliptique, la voix de Reynalda occupe tout de même beaucoup de place dans la conscience de Marie-Noëlle. Ainsi le récit de Nina (récit de 20 pages à la première personne) est juxtaposé au chapitre suivant (chapitre 6) à celui de Reynalda qui se fait sur 2 pages (pp. 207-209) et surgit d'un souvenir qui remonte à 8 ans plus tôt, à la veille du départ de Marie-Noëlle pour Boston².

Si à son arrivée à la Guadeloupe, l'enquête de Marie-Noëlle visait essentiellement la confirmation de ses conjectures sur l'identité de son père, il devient évident après le récit de Nina que «tout est à recommencer» (p. 207). Marie-Noëlle sera désormais tiraillée entre deux voix familiales affirmant deux «vérités» et deux versions opposées du passé:

Une pareille histoire ne s'invente pas. Pareils détails ne s'imaginent pas. Et pourtant l'une des deux femmes lui mentait avec aplomb. Laquelle? Est-ce que c'était Reynalda? Est-ce que c'était Nina? Elle ne saurait le dire et ainsi, elle n'aurait jamais la réponse à sa question. Cette idée-là l'avait mise au désespoir (p. 209).

(2) Ce même discours de Reynalda a été évoqué très brièvement à la p. 101 du roman. Mais il ne faut pas oublier que plus tôt dans le texte, MarieNoëlle se souvient aussi d'une autre conversation qu'elle a eue avec Reynalda quand Marie-Noëlle n'avait que 15 ou 16 ans et qu'on menaçait de la renvoyer de l'école. Voir le discours de Reynalda aux pages $62-70$ 
Il est évident, même dès l'incipit du texte, que le discours d'autrui travaille sans arrêt l'imagination de Marie-Noëlle: «Ranélise lui avait tant de fois raconté sa naissance qu'el croyait y avoir tenu un rôle; non pas celui d'un bébé terrorisé et passif [...] mais celui d'un témoin lucide; d'un acteur essentiel, voire de sa mère, l'accouchée, Reynalda elle-même qu'elle s'imaginait assise raide [...]» (p. 13).

Et ce que les autres ne lui racontent pas, Marie-Noëlle l'imagine et si bien qu'il est difficile de démêler ses vrais souvenirs de ceux qui sont inventés: «[1]e deuxième souvenir imaginaire de Marie-Noëlle, c'était celui de son baptême» (p. 17).

Si l'imagination de Marie-Noëlle colore la représentation des personnages ${ }^{3}$ et des événements, sa mémoire est forcément sélective: «Par contre, et Ranélise avait bien dû lui en faire le récit fréquemment, Marie-Noëlle ne gardait aucun souvenir du départ de sa mère» (p. 18). Pour Marie-Noëlle, la reconstitution de son histoire se fait donc par bribes et par fragments empruntés aux discours d'autrui, à des souvenirs réels et imaginés ainsi qu'aux lettres de Ludovic, de Garvey et d'autres - tous travaillés par l'imagination et par une mémoire incapable de saisir le réel dans son ensemble.

Le «silence» de Reynalda, ou plutôt sa version de la «vérité», est ainsi défié par les efforts de Marie-Noëlle pour reconstituer le passé de sa mère, alors que celleci avait vite fait de le rejeter pour «aller de l'avant». Aussi le silence de Reynalda s'oppose-t-il à plusieurs discours disparates, souvent contradictoires, effectués à des moments différents dans le temps et dans l'espace, à partir desquels Marie-Noëlle cherche à «déchiffrer l'indéchiffrable» (p. 34) afin de se composer une «histoire» unique et cohérente sur ses propres origines. Par leurs récits, plusieurs personnages participent à cette création: Ranélise, Reynalda, Nina, Ludovic, Garvey, Claire-Alta (la soeur de Ranélise), Bonne-Maman (la mère de Judes Anozie), Aristide Démonico (le mari de Fiorella) et Mme Marie Duparc (l'employeur parisien de la jeune Reynalda). À l'exception de Reynalda, de Nina et de Ludovic, le discours de ces personnages se manifeste principalement au style indirect ou au style narrativisé, ce qui implique leur prise en charge par une voix narrative régie.

Or, tout comme le récit de Nina s'oppose à celui de Reynalda, les paroles de ces divers personnages finissent tôt ou tard par révéler d'autres facettes de la réalité. Ainsi, Marie-Noëlle se souvient que d'après Ranélise, Reynalda est partie travailler en France grâce à l'intervention du BUMIDOM. Mais à la Guadeloupe, Claire-Alta lui révèle que Gérard Polius (l'amant de Ranélise) démentait cette histoire sachant, en tant que maire de la ville, que chaque candidat devait demander une enquête de bonnes moeurs de la mairie (p. 152). D'ailleurs, à Paris, Mme Marie Duparc dira que ce n'était pas le BUMIDOM mais un parent, l'évêque de la Guadeloupe, qui, à cette époque-là, les avait priés d'aider «une fille-mère de quinze ans, plus victime que pécheresse, plus malheureuse qu'endurcie, solitaire comme la solitude, affligée comme l'affliction» (p. 250). De plus, Aristide Démonico prétend qu'obsédée par la disparition inattendue de Reynada, Fiorella racontait à tous que son père, Gian Carlo Coppini avait l'habitude de violer Reynalda et que Nina Titane «lui aurai livré sa propre fille» (p. 173). Mais Aristide, le mari de Fiorella, n’y croit pas lui-même déduisant qu'il s'agissait d'«une banale affaire de jalousie filiale» (p. 173). Difficile de savoir la vérité... et la tâche que se donne Marie-Noëlle est justement de distinguer le vrai du faux afin de reconstituer correctement sa propre histoire: «Au fur et à mesure qu'elle parlait et répondait aux questions d'Anthea, elle mettait en place à sa manière les éléments obscurs du puzzle qui composaient sa vie» (p. 110).

Comme la déconstruction de l'histoire de Reynalda, la reconstitution de l'histoire de Marie-Noëlle se fait par le biais de voix multiples rappelant la technique

(3) Voir, par exemple, sa représentation du père Mondicelli p. 111. 
narrative déjà exploitée par Maryse Condé dans Traversée de la Mangrove et dans La Migration des coeurs. Reynalda, comme Francis Sanchez, est évidemment au centre de tous les discours. Mais la structuration du récit dans Desirada est bien plus complexe car, à la différence de Traversée dans la Mangrove, chaque chapitre du roman ne s'ouvre pas sur la voix d'un personnage distinct qui s'explique, (au discours direct pour les femmes ou indirect pour la plupart des hommes), plus ou moins au même moment que les autres comme lors de la veillée mortuaire de Francis Sanchez. Dans Desirada, ces discours sont articulés, comme on l'a vu, à des moments très disparates par des personnages vivant dans des lieux géographiques divers; et, plus important encore, ils sont rassemblés, assimilés, et peut-être même transformés, par une seule et même conscience - celle d'une jeune femme incapable d'affronter l'avenir tant qu'elle ne saura pas la vérité sur le passé.

Si dans une entrevue, Maryse Condé suggère au sujet de la polyphonie qu'elle est «toujours à la recherche d'une structure narrative qui épouse les ambiguïtés de [s]a pensée» ${ }^{4}$, elle affirme clairement, dans une autre, que l'emploi de la polyphonie lui permet de montrer que l'objectif de Marie-Noëlle est voué à l'échec:

Je crois que si nous pensons qu'il y a la réalité, la vérité, on n'arrive pas. Je crois que je suis arrivée à cette manière d'écrire parce que je pense que finalement il n'y a pas une vérité, il n'y a pas une image, il n'y a pas un centre, il n'y a pas une périphérie, il n'y a pas un 'je', il y a des ‘je' [...] je ne souhaite plus donner une seule vision des choses

Ainsi, la technique de la polyphonie traduit la coexistence de voix distinctes et de «vérités» contradictoires sans pour cela en privilégier une au détriment des autres ${ }^{6}$.

L'impossibilité de savoir de façon certaine la vérité fait de ce roman ce que Umberto Eco appellerait une «oeuvre ouverte». Se basant sur la notion que «l'oeuvre d'art est un message fondamentalement ambigu, une pluralité de signifiés qui coexistent en un seul signifiant», Eco cherche à montrer que «cette condition est propre à toute oeuvre d'art» et que «cette ambiguïté devient aujourd'hui une fin explicite de l'œuvre»'.

Au fond, une forme est esthétiquement valable justement dans la mesure où elle peut être envisagée et comprise selon des perspectives multiples, où elle manifeste une grande variété d'aspects et de résonances sans jamais cesser d'être elle-même. [...] En ce premier sens, toute oeuvre d'art, alors même qu'elle est forme achevée et 'close' dans sa perfection d'organisme exactement calibré, est 'ouverte' au moins en ce qu'elle peut être interprétée de différentes façons sans que son irréductible singularité en soit altérée 8 .

L'oeuvre ouverte est le «projet d'un message doté d'un large éventail de possibilités interprétatives»" Or l'oeuvre qui accentue son ambiguïté exige l'intervention

(4) Maryse Condé, Interview avec Suzanne Crosta: Plutôt que parler d'errance, je définirais ainsi la problématique de mon oeuvre. Elle se modèle sur mes réflexions, mes hésitations, mes recherches et mes solutions jamais définitives. D'autre part, je suis toujours à la recherche d'une structure narrative qui épouse les ambiguïtés de ma pensée «Exil, Migration, Ecriture: Une entrevue avec Maryse Condé» dans Récits de vie de l'Afrique et des Antilles, dir. Suzanne Crosta, Sainte-Foy, GRELCA, 1998, p. 123.

(5) Maryse Condé dans un entretien avec Maria Anagnostopoulou-Hielscher, Parcours identitaires de la femme antillaise, «Etudes francophones»,
1999, vol. XIV, n² 2, p. 77.

(6) Voir MikhaïL Bakhtine, Chapitre I, «Le Roman polyphonique» dans son essai La Poétique de Dostoievski, Paris, Editions du Seuil, 1970 (traduction française).

(7) Umberto Eco, L'Oeuvre ouverte, Paris, Editions du Seuil, 1965, pp. 9-10. Le terme «oeuvre» chez Eco désigne «un objet doté de propriétés structurales qui permettent, mais aussi coordonnent, la succession des interprétations, l'évolution des perspectives».

(8) Ibid., p. 17.

(9) Ibid., p. 11. 
active du lecteur. C'est une oeuvre «scriptible» et non «lisible» pour reprendre les termes de Roland Barthes. Maryse Condé semble approuver cette façon de lire:

Pour ce qui est du caractère provisoire du monde représenté dans Desirada, en effet, il n'y a pas de réponse car dans la vie nous n'avons pas de réponse, donc le récit est ouvert. Il revient à chaque lecteur de le recréer. On m'a fait la remarque que le livre est basé sur le mensonge. Pas du tout, l'histoire n'est pas un mensonge, il s'agit d'une pluralité d'interprétations que propose Reynalda. Ce qui lui est en fait arrivé n'est pas important en soi. Il s'agit que chacun de nous reconstruise l'histoire comme il veut ${ }^{10}$.

Comme Wolfgang Iser et d'autres critiques de l'herméneutique, Umberto Eco affirme que l'activité du lecteur est de plusieurs façons orientée par le texte:

Or, ici encore, «ouverture» ne signifie pas «indétermination» de la communication, «infinies» possibilités de la forme, liberté d'interprétation. Le lecteur a simplement à sa disposition un éventail de possibilités soigneusement déterminées, et conditionnées de façon que la réaction interprétative n'échappe jamais au contrôle de l'auteur ${ }^{11}$.

Dans Desirada, la liberté du lecteur est conditionnée, jusqu'à une certaine mesure, par la focalisation adoptée dans la narration. A première vue, il semble que le récit adopte un narrateur hétérodiégétique («à la troisième personne») dont la focalisation est largement déterminée par Marie-Noëlle (focalisation interne). Or la perspective de Marie-Noëlle n'est pas fixe mais subit constamment des modifications. Ainsi, par exemple, lors de son retour au pays natal, la Guadeloupe et la maison où MarieNoëlle a grandi diffèrent de celles de ses souvenirs (p. 139); à Paris, l'appartement de Mme Duparc et Mme Duparc elle-même ne sont pas du tout comme Marie-Noëlle se les a représentés (p. 247). D'ailleurs, ses convictions négatives à l'égard des hommes s'effritent à la rencontre de Ludovic pour se confirmer plus tard après le récit de celui-ci: «ce n'était qu'un coureur comme les autres» (p. 280). De même, Marie-Noëlle semble prêter foi au récit de Reynalda jusqu'à ce qu'elle entende le récit de Nina après quoi elle n'est même plus sûre de rien: «Marie-Noëlle n'arrivait pas à douter de la sincérité de sa grand-mère. Une intuition lui soufflait que Nina disait la vérité. Puis, elle mettait en doute cette intuition, la repoussait, puisqu'elle n'avait qu'une seule cause: la rancoeur qu'elle garderait toujours à l'endroit de Reynalda» (p. 214). Comme Marie-Noëlle, le lecteur se trouve face à différentes interprétations possibles des mêmes événements et est, lui aussi, dans l'impossibilité de résoudre le problème. La polyphonie permet à Maryse Condé d'évoquer et de soutenir cette ambiguiité:

To create a sorte of polyphonie, I introduce a voice which is not the narrator's voice, a voice that subverts the narrative that the author, Maryse Condé is presenting. The récits and the voices are there to create a sort of contradictory polyphony so that the reader can form his own opinion. I don't think the author, the narrator, should always provide the definitive version $^{12}$.

Bien qu'étant le sujet de la focalisation et la mémoire permettant l'intégration des discours d'autrui, Marie-Noëlle ne relate son récit à la première personne que dans le dernier chapitre du roman. Cela est très significatif dans la mesure où Ma-

(10) Maryse Condé et Marie-Agnès Sourieau, Entretien avec Maryse Condé: de l'identité culturelle, «The French Review», vol. 72, n 6, Mai 1999 , p. 1096.

(11) Umberto Eco, L'Oeuvre ouverte, Paris, Edi- tions du Seuil, 1965, p. 19

(12) Maryse Condé, entrevue avec Robert H. McCormick, Desirada - a new conception of identity, «World Literature Today», vol. 74, n 3, Été 2000 , p. 520. 
rie-Noelle se dit incapable d'agir et de prendre la parole tant qu'elle n'aura pas fait éclater le silence de sa mère. Sa prise de parole dans le dernier chapitre du roman révèle que Marie-Noelle est effectivement la génératrice de l'ensemble du récit et cela en dépit de l'usage de la troisième personne. Elle s'invente une histoire et elle en est le scribe. Et par cet acte, elle se venge du silence (et du 'mensonge') de sa mère et réussit pour la première fois de sa vie à lui emboîter le pas. Marie-Noëlle semble finalement mettre en pratique les conseils de sa mère Reynalda (p. 62), de sa grand-mère Nina (p. 202) et de Ludovic: «Je te répète, si j'ai un conseil pour toi, c'est d'aller enfin de l'avant. Reynalda te donne l'exemple. Elle te prouve que le passé, même le plus douloureux, finit par mourir et que la passion réalise les ambitions qui semblent aux autres les plus extravagantes» (p. 278). C'est à la fin du texte, lorsque Marie-Noëlle devient finalement la narratrice de son histoire, que sa réconciliation avec elle-même et que les rapports entre sa quête et l'écriture deviennent évidents.

L'écriture pour inventer des vies

«Honteuse, je me tairai donc en attendant qu'à mon tour j'apprenne à inventer des vies». (p. 281)

Dans Desirada, l'écriture est un thème récurrent qui se manifeste implicitement par le biais de l'intertextualité (fréquente chez Maryse Condé) et par l'intermédiaire de personnages impliqués dans la production de thèses, de lettres, etc. Il y a dans ce texte plusieurs renvois à des oeuvres littéraires très connues. On remarque une référence à un poème de Rimbaud dans la phrase: «la nuit où il dérivait sur le fleuve comme un bateau ivre» (p. 161); à une pièce d'Ionesco dans la phrase: «elle avait demandé inquiète si une cantatrice noire n'est pas plus déplacée qu'une cantatrice chauve» (p. 234); à un poème d'Apollinaire dans la phrase: «Sous le pont Mirabeau, coule toujours la Seine» (p. 238); à un roman de Jacques Roumain dans: «Moi, j’étais devenu le gouverneur de la rosée. Moi, j'avais trouvé l'eau» (Ludovic p. 275); et finalement à un roman de Lewis Carroll dans la phrase: «Imagination! Imagination!» (p. 247). Ce sont autant de clins d'oeil au lecteur faisant ressortir le savoir culturel et littéraire des protagonistes.

Dans Desirada, le thème de l'écriture se manifeste surtout par le biais de personnages féminins qui ont écrit ou écrivent des textes documentaires: essais sociologiques et thèses de doctorat. Ainsi Reynalda a écrit une thèse et elle a publié Les Jours étrangers, un ouvrage sur les migrants originaires des Antilles et de l'Afrique sub-saharienne (p. 220). Anthea Jackson a rédigé une thèse sur Jane Austen avant de se consacrer aux récits de femmes esclaves du début du XIX ${ }^{\mathrm{e}}$ siècle (p. 109). MarieNoëlle écrit une thèse sur Jean Genet qui, en classe, «était prétexte à des joutes sans fin sur l'exclusion, le colonialisme, le vol, la prison, l'amour, le sexe, la virilité qu'on aurait pu juger bien éloignées de la littérature» (p. 142).

Or ces écrits sont en l'occurrence les premiers pas vers un autre type d'écriture qu'elle soit qualifiée d'«autobiographique» ou de «fictive», peu importe:

Après Les Jours étrangers, [Reynalda] m'a annoncé qu'elle allait publier un roman, l'autobiographie à laquelle elle travaille depuis que je la connais [...] Ainsi, elle se libérera une fois pour toutes de la vérité. Pourtant, la connaissant comme je la connais, je sais que cette véritélà sera une fiction. D'ailleurs, qu'est-ce que nous pouvons construire quand nous parlons de nous-même? (p. 278). 
Il importe ici de constater que Marie-Noëlle aussi caresse l'espoir d'écrire autre chose qu'une thèse; mais elle doit tout d'abord affronter deux difficultés: d'une part, l'impossibilité d'affirmer son identité et, d'autre part, le sentiment de son infériorité face aux diverses réussites de sa mère.

Sa thèse lui fournissait un bon prétexte pour refouler ses confuses velléités de devenir un écrivain. Au fond d'elle-même, elle n'était pas dupe. Comment pouvait-elle écrire? Comment pouvait-elle prendre la plume tant qu'elle ne saurait ni qui elle était ni d'où elle sortait? Bâtarde née de père inconnu. Belle identité que celle-là! Tant qu'elle n'aurait pas d'autres indications à inscrire sur son livret de famille, elle ne pourrait rien mener à terme. Bientôt, elle se donna un deuxième bon prétexte. Peu avant Noël, Ludovic lui adressa Les Jours étrangers, ouvrage que venait de publier Reynalda. [...] Regardant le nom qui s'étalait en couverture: Reynalda Titane, six syllabes obscures, en apparence anodines, pas très élégantes ni euphoniques, en réalité chargées du pouvoir de blesser et de mal faire, il lui sembla que sa maman lui livrait une lutte sans merci. Qu'elle s'ingéniait à lui barrer toutes les issues de secours possibles. Elle lui avait barré l'amour, la maternité. A présent, elle lui barrait l'écriture (p. 220).

Nonobstant ces obstacles, il devient assez clair que le récit que nous lisons est engendré par la fille et non pas par la mère. A force de suivre Marie-Noëlle dans sa quête de la vérité, à force d'embrasser sa vision tout le long du récit et, finalement, à force d'entendre sa voix, il est facile de déduire que «l'auteur» (fictif) du récit est Marie-Noëlle qui, après avoir subi une dépression, réussit enfin à se forger une histoire et une identité nonobstant son souci de ne pas blesser sa mère.

Voilà que cette histoire des origines, cette histoire qui ne reposait que sur le dire, la parole des uns et des autres, rentrait dans la réalité. Elle n'attendait plus que la main d'un scribe pour la fixer par écrit et ainsi lui donner la pesanteur de la vérité. [...] Alors qu'elle n'avait aucun droit sur cette histoire. Elle ne lui appartenait que parce qu'elle appartenait à Reynalda. Reynalda qui s'était enveloppée de silence qui avait rompu tous les liens qui l'amarraient à l'île. Est-ce qu'écrire ne reviendrait pas à la trahir, à la blesser encore une fois? (p. 171).

Ainsi Marie-Noëlle trouve son salut personnel dans la création d'une histoire ${ }^{13}$ qui n'est fondée exclusivement ni sur le dire de Reynalda ni sur le dire des autres personnages.

Je suis sortie du noir. Je suis sortie mal armée, pas du tout parée pour l'existence de la tête brillante de ma mère. Ce n'est pas la peine de la confronter. Elle ne démordra pas de son histoire. D'ailleurs, je suis sûre qu'elle a fini par y croire. [...] Dans le fond, elle est un écrivain, ma mère, et elle a bâti sa fiction. Moi qui vis, je dois chercher la vérité autre part. Où? Il faudrait tout ré interpréter, tout recommencer depuis le commencement (p. 252).

Ainsi à partir des 'pièces d'un puzzle vieux de trente ans', Marie-Noëlle arrive à créer sa propre version des événements et donc une nouvelle histoire qu'elle oppose discrètement à celle de sa mère: «Elle s'apercevait aussi qu'il manquait un chapitre, des chapitres à l'histoire qu'elle amassait en elle. Il manquait les chapitres du milieu, car Reynalda n'avait jamais parlé que du commencement. De son enfance. Comme si seul ce temps-là comptait pour elle» (p. 165). Son entrevue avec Mme Duparc lui révèle «une faille dans l'édifice de granit que Reynalda avait construit» et à partir de laquelle «la vérité, pourrait peut-être se faire jour» (p. 250).

(13) Dans son entrevue avec Robert McCormick, Maryse Condé observe: «When you try to tell the truth about your life, you realize that your truth is fiction and that you are fabricating a reality, a somewhat imaginary life». «World Literature Today», vol. $74, n^{\circ} 3$, Été 2000 , p. 525. 
C'est surtout à ce moment-là qu'une lecture rétrospective permettrait d'entrevoir que peut-être après tout, le père de Marie-Noëlle n'est pas Gian Carlo Coppini mais un homme d'église: si ce n'est pas l'évêque qui dans sa lettre à Mme Duparc se désole d'avoir incité le déplacement de Nina et de sa fille à La Pointe et dont la présence insolite chez les Coppini aurait été fortement commentée, c'est peut-être le père Mondicelli, le confesseur d'Arcania, constamment présent chez Gian Carlo Coppini, et qui quitte La Pointe pour travailler chez les lépreux (p. 198) peu après la disparition de Reynalda.

Comment est-ce qu'elle n'avait pas compris cela plus tôt? Tout s'emboîtait. Toutes les bribes éparpillées du puzzle se mettaient en place. La confession. La musique. Cherubino. Voi che sapete che cosa è amor. Mon enfant, comme tu as une jolie voix. Je te ferai partir étudier en métropole. Tu seras paggio sur la scène de l'Opéra. Tu as une jolie voix et de beaux yeux aussi, tu sais. Approche-toi plus près. Encore plus près... Ah! Qu'est-ce que j'ai fait? Périsse le jour où je suis né! [...] Mon père, tu ne pourras jamais au grand jamais me pardonner. Désormais, je vais rejoindre ceux qui me ressemblent. Les pustules de leur corps couvrent mon âme (p. 256).

Douée d'une voix de mezzo-soprano magnifique (p. 233), Reynalda avait attiré l'attention de l'évêque de la Guadeloupe. Et comme l'adolescente caressait l'ambition de devenir cantatrice, on peut en déduire que sa grossesse était le résultat d'une séduction et non d'un viol. Cette déduction s'appuie non seulement sur la jalousie de Reynalda pour un missel - l'unique objet qu'elle refuse de partager - mais aussi sur son manque de retenue, fait plutôt inhabituel chez elle, quand elle évoque «une personne» lui ayant promis une carrière musicale: «C'était la seule fois où Garvey l'avait vue manifester une émotion et c'était à l'occasion d'un vaniteux songe de grandeur, d'un égoïste apitoiement sur elle-même» (p. 234).

Marie-Noëlle défie la «fiction» de sa mère en suggérant une autre version des événements. Elle le fait en sachant que son salut personnel en dépend. Mais elle reste discrète, sensible, peut-être toujours soucieuse à l'égard de sa mère, refusant d'imposer une seule version des faits (la séduction) au détriment de l'autre (le viol). Comme Maryse Condé, Marie-Noëlle entretient l'ambiguïté, le doute permettant la co-existence de plusieurs «vérités». Pour cette raison, nous ne saurons pas conclure, en toute simplicité, que le père de Marie-Noëlle était le père Mondicelli14 ${ }^{14}$ La question n'est pas là: elle réside plutôt dans ce que le texte nous révèle au sujet du passé et de l'identité par le biais de la polyphonie et par sa genèse de l'ambiguïté ${ }^{15}$.

$\mathrm{Si}$, au début de sa quête, Marie-Noëlle vise la certitude d'une identité sûre, stable, fixée dans l'identité de ses parents, dans un seul espace et dans un seul pays, elle en vient à douter de cette nécessité:

Est-ce qu'elle ne pouvait pas continuer de vivre comme elle le faisait? Sans identité, comme une personne à qui on a volé ses papiers et qui erre à travers le monde? Est-ce qu'ainsi elle n'était pas plus libre? C'est une sale manie de vouloir savoir à tout prix d'où on sort et la goutte de sperme à laquelle elle doit la vie (p. 243).

A la fin de sa quête, Marie-Noëlle revêt de plein gré sa «monstruosité»: «réelle ou imaginaire, cette identité-là avait fini par me plaire. D'une certaine manière, ma

(14) Voir à ce propos Maryse Condé: 'Désirada' ou l'ironie du sort, de Marianne Bosshardt in Maryse Condé: Une nomade inconvenante, IBIS Rouge Editions, 2002, p. 156.

(15) Dans son étude de La vie scélérate, Dominique Licops affirme que le «récit polyphonique refuse de donner plus de légitimité à une identité plutôt qu'à une autre». Voir Expériences diasporiques et migratoires des villes dans 'La vie scélérate' et 'Desirada' de Maryse Condé, «Nottingham French Studies», vol. 39, n 1, Printemps 2000, p. 114. 
monstruosité me rend unique. Grâce à elle, je ne possède ni nationalité ni pays ni langue» (p. 281). Nous sommes d'accord avec Dominique Licops qui affirme: «En fait, avec ce roman, Condé remet en question la famille et la généologie comme principes identitaires et passe d'une définition plus inclusive de l'identité à une identité dont le manque de définition par le passé et la généalogie est caractéristique» ${ }^{16}$.

Et pour conclure cette étude de la polyphonie et de l'écriture dans Desirada, citons un passage de Maryse Condé qui affirme, sans ambiguïté, que la quête identitaire de Marie-Noëlle est à l'image même de celle des Antilles - sujet, sans doute, d'un grand intérêt pour une analyse future:

Mais, finalement la leçon c'est qu'il faut qu'elle apprenne à vivre sans savoir qui est son père, sans avoir de pays bien à elle (ce n'est ni la Guadeloupe ni la France ni l'Amérique), la leçon c'est que les Antilles ont assez posé de questions sur leur passé, sur leur identité, maintenant même si on ne peut pas tout à fait répondre à ces questions il faut accepter de vivre comme on est. C'est un peu optimiste.

[...] pendant trop longtemps nous avons cherché l'origine, on a pensé que sans savoir d'où l'on venait on ne pouvait pas arriver à être équilibrés [...] maintenant, puisque c'est tellement difficile et qu'on n'y arrive jamais, je crois qu'il faut prendre son parti et vivre avec ces lacunes, avec ces absences ${ }^{17}$.

ANNA MARIA MIRAGLIA

(16) Dominique Licops, Expériences diasporiques et migratoires des villes dans 'La vie scélérate' et 'Desirada' de Maryse Condé, «Nottingham French Studies», vol. 39, $\mathrm{n}^{\circ} 1$, Printemps 2000 , p. 115.
(17) Voir l'entrevue accordée à Maria Anagnostopoulou-Hielscher, Parcours identitaires de la femme antillaise, «Etudes francophones», 1999, vol. XIV, n 2 2, p. 77. 